Research Paper

\title{
Glucose Transporter 1 Promotes the Malignant Phenotype of Non-Small Cell Lung Cancer through Integrin $\beta 1 /$ Src/FAK Signaling
}

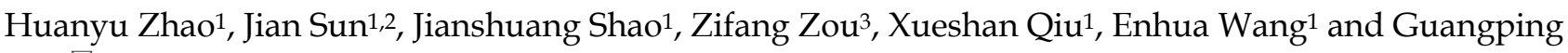 \\ $\mathrm{Wu}^{1 \bowtie}$ \\ 1. Department of Pathology, The First Affiliated Hospital and College of Basic Medical Sciences, China Medical University, No. 155 Nanjing North Street, \\ Heping District, Shenyang, 110001, China \\ 2. Guangzhou DaAn Clinical Laboratory Center, No. 74 Zhongshan Er Road, Guangzhou, 510000, China \\ 3. Department of Chest Surgery, The First Affiliated Hospital, China Medical University, No. 155 Nanjing North Street, Heping District, Shenyang, 110001, \\ China
}

$\square$ Corresponding author: Professor Guangping Wu, Department of Pathology, The First Affiliated Hospital and College of Basic Medical Sciences, China Medical University; No. 155 Nanjing North Street, Heping District, Shenyang, 110001, China; Tel: 86-24-83282177; Fax: 86-24-83282177; E-mail: wugp@cmu1h.com.

(c) The author(s). This is an open access article distributed under the terms of the Creative Commons Attribution License (https://creativecommons.org/licenses/by/4.0/). See http:/ /ivyspring.com/terms for full terms and conditions.

Received: 2018.10.18; Accepted: 2019.06.23; Published: 2019.08.27

\begin{abstract}
Background: Glucose transporter 1 (GLUT1) is the main factor of Warburg effect, which is associated with poor prognosis in many tumors. However, the underlying molecular mechanism of GLUT1 in the progression of non-small cell lung cancer (NSCLC) is unclear.

Methods: We used quantitative real-time PCR to detect GLUT1 mRNA expression in bronchial brushing samples and performed Western Blot and biological behavior testing to check the effect of GLUTI on NSCLC cell proliferation, migration, invasion and apoptosis.

Results: We found that the $C(t)$ normalized value of GLUT1 in malignant bronchial brushing samples was significantly higher than that in benign samples $(P<0.05)$. GLUT1 significantly increased the expressions of cyclin A, cyclin D1, cyclin E, cyclin dependent kinase 2 (CDK2), CDK4, CDK6 and matrix metalloproteinase 2 (MMP2), but decreased the expressions of p53 and p130 in NSCLC cells. The biological behavior testing indicated that GLUT1 enhanced NSCLC cell proliferation, invasion and migration but inhibited cell apoptosis. In addition, GLUT1 upregulated the expression of integrin $\beta 1$ and promoted the phosphorylation of focal adhesion kinase (FAK, phosphorylation at Tyr576/577) and Src (Src phosphorylation at Tyr530). siRNA knock down of integrin $\beta 1$ expression suppressed GLUT1 induced NSCLC cell biological behavior, as well as the phosphorylation of FAK and Src.

Conclusion: Taken together, our data confirms that GLUT1 promotes the malignant phenotype of NSCLC through integrin $\beta 1 / \mathrm{Src} / \mathrm{FAK}$ signaling, which provides a new therapeutic target for the treatment and research of lung cancer.
\end{abstract}

Key words: Glucose transporter 1; non-small cell lung cancer; integrin $\beta 1$; focal adhesion kinase; Src

\section{Introduction}

Lung cancer is correlated with high morbidity and mortality. Early diagnosis of lung cancer can improve patient's cure rate. Bronchial biopsy is a method for diagnosing lung cancer. And bronchial brushing is an important supplement for bronchial biopsy. When biopsy cannot be performed (for example, lesions of the distal bronchus or if surface ulceration is severe), analysis of bronchial brushing cells is particularly important. Compared with homologous normal cells, malignant tumor cells have different metabolic pathways [1, 2]. In aerobic conditions, glycolysis will consume more glucose to gain energy. It is called Warburg effect, which is associated with tumor progression [3]. In addition to 
Warburg effect, glucose transporter (GLUT) facilitates glucose uptake on plasma membrane. Glucose uptake is important for tumorigenesis [4]. Different members in the GLUT family are confirmed to be upregulated in malignant tumor. GLUT1 is the main factor of Warburg effect, which is the main carrier of glucose transmembrane transport [5]. GLUT1 overexpression is associated with poor prognosis in many types of tumors, including lung cancer $[6,7,8]$. This brings us to the idea that GLUT1 might take part in the progression of NSCLC.

MMPs are the most important enzyme for degradation of extracellular matrix (ECM). As a member of MMPs, MMP2 is widely regarded as a marker of tumor invasion and metastasis. It could significantly hydrolyze collagen I, which is the most important component of basement membrane $[9,10]$. In rhabdomyosarcoma cell line, GLUT1 regulates the expression of MMP2, as well as the interaction of p53 with the MMP2 promoter [11]. However, it is not clear that whether there is an association between GLUT1 and MMP2 in NSCLC.

As a family of cell surface receptors, integrin is a heterodimeric transmembrane receptor formed by non-covalently bound $\alpha$ and $\beta$ subunits. It mediated adhesion to the ECM [12]. Focal adhesion kinase (FAK) is a non-receptor kinase that controls the migration, proliferation, and survival of cells [13-15]. FAK can be activated by the interaction between ECM and integrin [16]. GLUT1 knockdown inhibited cell proliferation, invasion, and migration through integrin $\beta 1 / \mathrm{Src} / \mathrm{FAK}$ signaling pathways in breast cancer [17]. We have performed RT-PCR to test GLUT1 expression and found that it was overexpressed in bronchial brushing samples of NSCLC patients [18]. However, it is not clear whether GLUT1 is correlated with the progression of NSCLC.

In this study, we used quantitative real-time PCR to detect GLUT1 expression in bronchial brushing samples and performed biological behavior testing to check the effect of GLUT1 on NSCLC cell proliferation, migration, invasion and apoptosis so as to reveal its clinical value in the early diagnosis and treatment of lung cancer.

\section{Materials and methods}

\section{Patients and specimens}

We collected 104 bronchial brushing samples from the patients who did not underwent surgery in the First Affiliated Hospital of China Medical University from December 2014 to February 2015. There were 64 malignant samples (lung squamous cell carcinoma: 40 cases; lung adenocarcinoma: 24 cases) and 40 benign samples (pneumonia: 37 cases; tuberculosis: 3 cases). Cytology samples obtained from the bronchial brushing and the transbronchial needle aspiration (TBNA). All samples were obtained in accordance with Human Subject Research Protocols approved by the ethics committees of the First Affiliated Hospital of China Medical University (No.AF-SOP-07-1.0-01).

\section{Cell culture}

The NSCLC cell lines (human lung adenocarcinoma cell line: H1299, SPC and A549; squamous cell carcinoma cell line: LK2 and SK-MES-1) and HBE (human bronchial epithelial cell line) cell. These cells were purchased from the American Type Culture Collection (Manassas, VA, USA). They were cultured in the medium of RPMI 1640 (Invitrogen, Carlsbad, CA, USA) or DMEM (Invitrogen, Carlsbad, CA, USA) including 10\% fetal bovine serum (FBS). We detected GLUT1 protein expression in NSCLC cell lines and compared it with HBE (Figure S1). We selected A549 (human lung adenocarcinoma cell line, GLUT1 high expression) and LK2 (human squamous cell carcinoma cell line, GLUT1 low expression) for subsequent experiment.

\section{Western Blot}

Total protein from NSCLC cell lines was extracted in lysis buffer and quantified by the method of Bradford. Extracted protein (Fifty micrograms) was separated by SDS-PAGE, and then transferred to polyvinylidene fluoride membranes. The membranes were blocked with 5\% skim milk. After incubating overnight $\left(4^{\circ} \mathrm{C}\right)$ with primary antibodies, the membranes were incubated with HRP-labeled secondary antibody for 2 hours at room temperature. Protein bands were examined with BioImaging System through ECL staining. We selected GAPDH as reference. The primary antibodies used in this study are shown in Table S1.

\section{Transfection}

GLUT1 siRNA and control siRNA were purchased from Santa Cruz Biotechnology (CA, USA; catalog number: sc-35493), integrin $\beta 1$ siRNA and control siRNA were purchased from Santa Cruz Biotechnology (CA, USA; catalog number: sc-35674), and GLUT1 plasmid were purchased from OriGene (Rockville, USA; catalog number: SC116011). They were transiently transfected to cell line with Lipofectamine 2000 (Invitrogen, Carlsbad, USA; catalog number: 11668030) according to the instruction of manufacturer.

\section{Quantitative real-time PCR}

Quantitative real-time PCR was performed by GoTaq ${ }^{\circledR}$ qPCR Master Mix (Promega, USA) according 
to the instruction of manufacturer. We used 7900HT Fast Real-Time PCR System (Applied Biosystems). The $C(t)$ value was normalized using $\beta$-actin. Each experiment was repeated by three times. The primers' sequences used in this study are as follows: GLUT1 Fwd 5'-CTGGCATCAACGCTGTCTTC-3', Rev 5'-GCCTATGAGGTGCAGGGTC-3'; $\beta$-actin Fwd 5'-GTCCACCTTCCAGCAGATGTG-3', Rev 5'-GCAT TTGCGGTGGACGAT-3'.

\section{Transwell assay}

We used transwell membranes $(8 \mu \mathrm{m}$ pore polycarbonate membrane) in 24-well plates and applied Matrigel (BD Bioscience) to the upper surface of the membrane in each well. Cells (treating with different factor, $3 \times 10^{5}$ cells in $100 \mu \mathrm{l}$ serum-free medium) were cultured in the upper chamber, and the medium containing 10\% FBS was added to the lower chamber. After incubation for 20h, the cells were stained with hematoxylin (Sigma). For each filter, we randomly selected 10 fields (400×magnification) under microscope to count the number of invaded cells.

\section{Cell scratch experiment}

Cells were seeded in 6-well plates, and after being treated with each factor, each well was scratched with a $100 \mu l$ pipette tip. The width of scratch was examined under the microscope at the time points of 1 hour and 24 hours at 3 separated sites.

\section{Apoptosis assay (flow cytometry)}

After various treatments, cells were harvested and washed twice with phosphate-buffered saline (PBS) by gentle shaking. Binding buffer $(500 \mu l)$ was added to resuspend cells, next Annexin V-FITC (5 $\mu 1)$

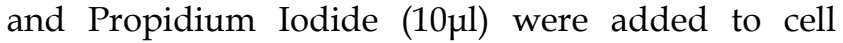
suspension Mix, and then darkly incubated for 15 minutes at room temperature until the detection by flow cytometry (Becton-Dickinson, San Jose, CA, USA).

\section{Colony formation assay}

Cells (treating with different factors) were seeded in $6 \mathrm{~cm}$ dishes (1000 cells/dish) and cultured for 12 days. The plates were washed with PBS and stained with Giemsa. We counted the number of colonies (each with more than 50 cells) by microscopic Q.Z.D.

\section{Statistical analysis}

We performed SPSS 17.0 for all of above analyses. All datas were reported as mean $\pm S D$, which was analyzed based on the Student's t-test. A two-tailed $P<0.05$ was considered to be statistically significant.

\section{Results}

\section{GLUT1 mRNA expression in bronchial brushing samples}

We used quantitative real-time PCR to detect bronchial brushing samples and made the standard curve for GLUT1 (target gene) and $\beta$-actin (reference gene) (Figure S2). GLUT1 $\mathrm{C}(\mathrm{t})$ normalization values (mean \pm SD) in benign group and malignant group (adenocarcinoma group and squamous cell carcinoma group) were detected (Table 1). GLUT1 C(t) normalized value in malignant group was significantly higher than that in benign group $(P<0.05)$.

Table 1. The $C(t)$ normalization value (mean $\pm S D$ ) of GLUT1 in bronchial brushing samples.

\begin{tabular}{lllll}
\hline Group & $\begin{array}{l}\text { Benign } \\
(\mathrm{n}=40)\end{array}$ & $\begin{array}{l}\text { Malignant } \\
(\mathrm{n}=64)\end{array}$ & $\begin{array}{l}\mathrm{SCC} \\
(\mathrm{n}=40)\end{array}$ & $\begin{array}{l}\text { ADE } \\
(\mathrm{n}=24)\end{array}$ \\
\hline $\begin{array}{l}\text { GLUT1 C }(\mathrm{t}) \\
\text { normalization value }\end{array}$ & $0.15 \pm 0.03$ & $0.85 \pm 0.19^{*}$ & $1.18 \pm 0.29^{*}$ & $0.31 \pm 0.07^{*}$ \\
\hline
\end{tabular}

* $P<0.05$ compared with benign group; SCC, squamous cell carcinoma; ADE adenocarcinoma.

\section{The effect of GLUT1 to NSCLC cell proliferation and cell cycle}

Previous study confirmed that Ghrelin enhanced GLUT1 expression and promoted oral cancer cell proliferation [19]. GLUT1 overexpression promoted cell viability and proliferation [20]. Above reports reminded us that there might be a relationship between GLUT1 and NSCLC cell proliferation. Based on GLUT1 protein expression in NSCLC cell lines (Figure S1), we selected A549 (GLUT1 high expression) and LK2 (GLUT1 low expression) for subsequent experiment. We transfected siRNA-GLUT1 to A549 and GLUT1 expression plasmid to LK2 cell line. The result of MTT and colony formation assays showed that siRNA-GLUT1 significantly inhibited cell colony formation and proliferation in A549 cell and GLUT1 transfection had the opposite effect in LK2 cell $(P<0.05$, Figure 1).

For detecting the effect of GLUT1 to cell cycle, we performed Western Blot to test the cell cycle correlated proteins (cyclin A, cyclin D1, cyclin E, CDK2, CDK4, CDK6, p21, p53, pRB, p130). The result showed that adding siRNA-GLUT1 to A549 cell significantly decreased the expressions of cyclin A, cyclin D1, cyclin E, CDK2, CDK4, CDK6 and increased the expressions of p53 and p130, but had no effect on p21 and pRB (Figure 2). GLUT1 transfection had the opposite effect in LK2 cell. These results indicated that GLUT1 could regulated cell cycle of NSCLC cells. 

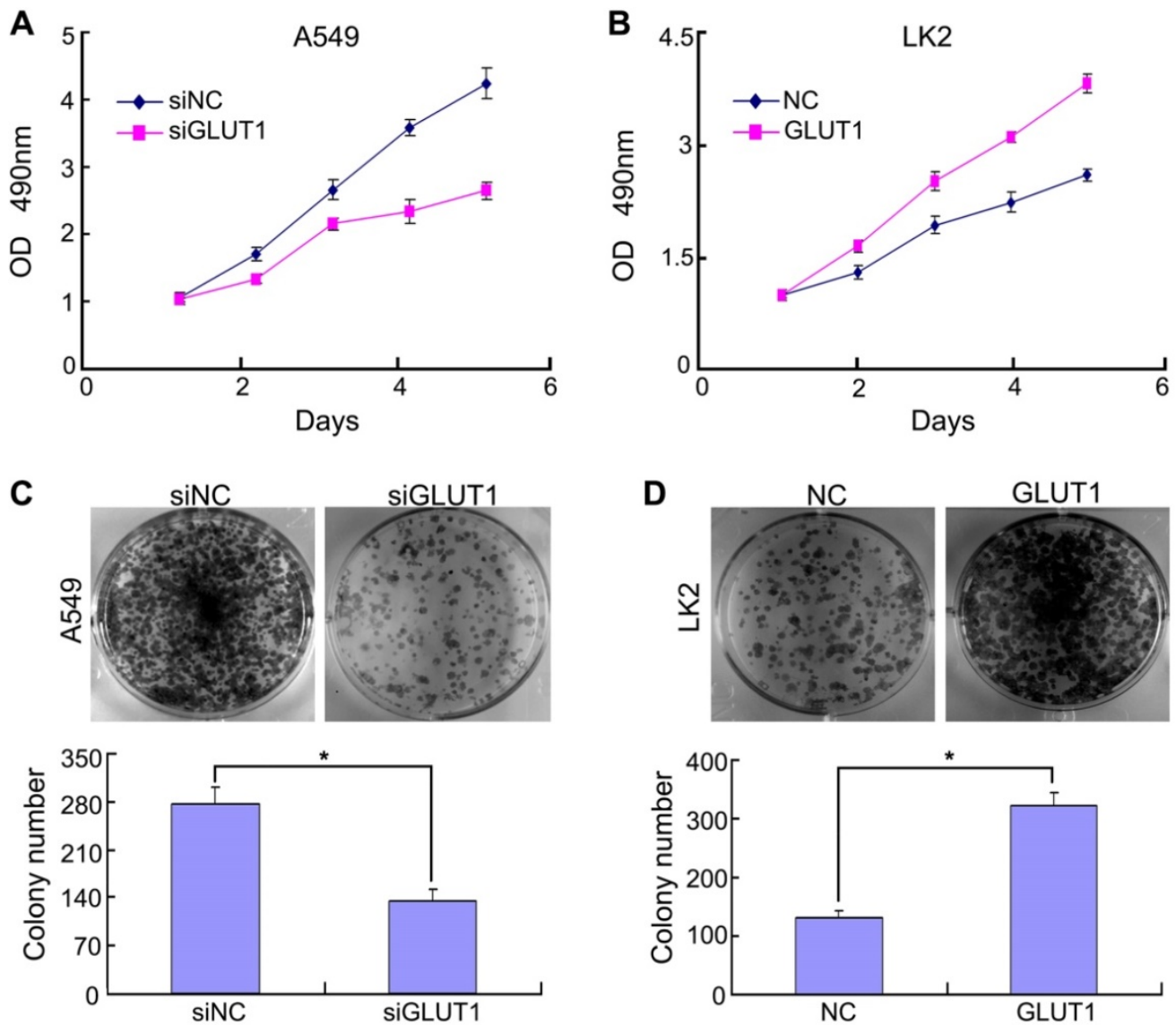

Figure 1. The effect of GLUT1 on NSCLC cell proliferation and colony formation. (A, B) NSCLC cell proliferation; (C, D) NSCLC cell colony formation. Adding siRNA-GLUT1 (siGLUT1) to A549 cell and GLUT1 expression plasmid (GLUT1) to LK2 cell; the graph in (C) and (D) shows the number of colony formation under different treatments; *, $P$ $<0.05$ compared with negative control; NC, negative control; si-NC, scramble siRNA for negative control; Error bars, S.D.

\section{The effect of GLUT1 to cell migration of NSCLC cell}

We used cell scratch to detect the effect of siRNA-GLUT1 and GLUT expression plasmid to on NSCLC cell migration. The results showed that siRNA-GLUT1 inhibited cell migration in A549 cell and GLUT1 transfection had the opposite effect in LK2 cell $(P<0.05$, Figure 3A and 3B).

The result of Western Blot showed that siRNA-GLUT1 significantly inhibited the expression of Rho-associated coiled-coil containing kinase 1 (ROCK1) and ROCK2 in A549 cell, and GLUT1 had the opposite effect in LK2 cell. However, siRNA-GLUT1 or GLUT1 transfection had no effect on RhoA expression (Figure 3C and 3D).

\section{The effect of GLUT1 to cell invasion of NSCLC cell}

We used Transwell to detect the effect of siRNA-GLUT1 and GLUT1 expression plasmid on NSCLC cell invasion. The results showed that adding siRNA-GLUT1 inhibited cell invasion in A549 cell and GLUT1 transfection had the opposite effect in LK2 $(P<0.05$, Figure 4A and 4B).
Tissue inhibitor of matrix metalloproteinase 2 (TIMP2) is a inhibitor of MMP2. The balance between MMP2 and TIMP2 plays a key role in tumor cell invasion and metastasis [21]. proMMP2/MMP2 activation pathway is critical for cancer cell metastasis [22]. The result of Western Blot showed that adding siRNA-GLUT1 significantly inhibited the expression of MMP2 and proMMP2 in A549 cell, and GLUT1 transfection had the opposite effect in LK2 cell. The result indicated that GLUT1 had a positive effect on the expressions of MMP2 and proMMP2 in NSCLC cells. Meanwhile, GLUT1 had negative effect on TIMP2 expression (Figure 4C and 4D).

\section{The effect of GLUT1 to NSCLC cell apoptosis}

We transiently transfected siRNA-GLUT1 and GLUT1 expression plasmid to NSCLC cells. Annexin $\mathrm{V}$ staining assay was used to determine the effect of GLUT1 on apoptosis of A549 and LK2 cells. The apoptosis rate was markedly increased after siRNA-GLUT1 transfection in A549 cell, but significantly decreased after GLUT1 transfection in LK2 cell $(P<0.05$, Figure 5A and 5B). The results indicated that GLUT1 could inhibit the apoptosis of NSCLC cells. 


\section{GLUT1 regulates NSCLC cell proliferation, migration, invasion and apoptosis through integrin $\beta 1 /$ Src/FAK signaling pathway}

Integrin could regulate many biological processes, including cell migration and proliferation [23-25]. Integrin has not catalytic activity, but it can bind and activate signaling proteins, such as non-receptor tyrosine kinase, including FAK and Src [26]. The association between FAK and integrin $\beta 1$ is required for FAK activation [27].

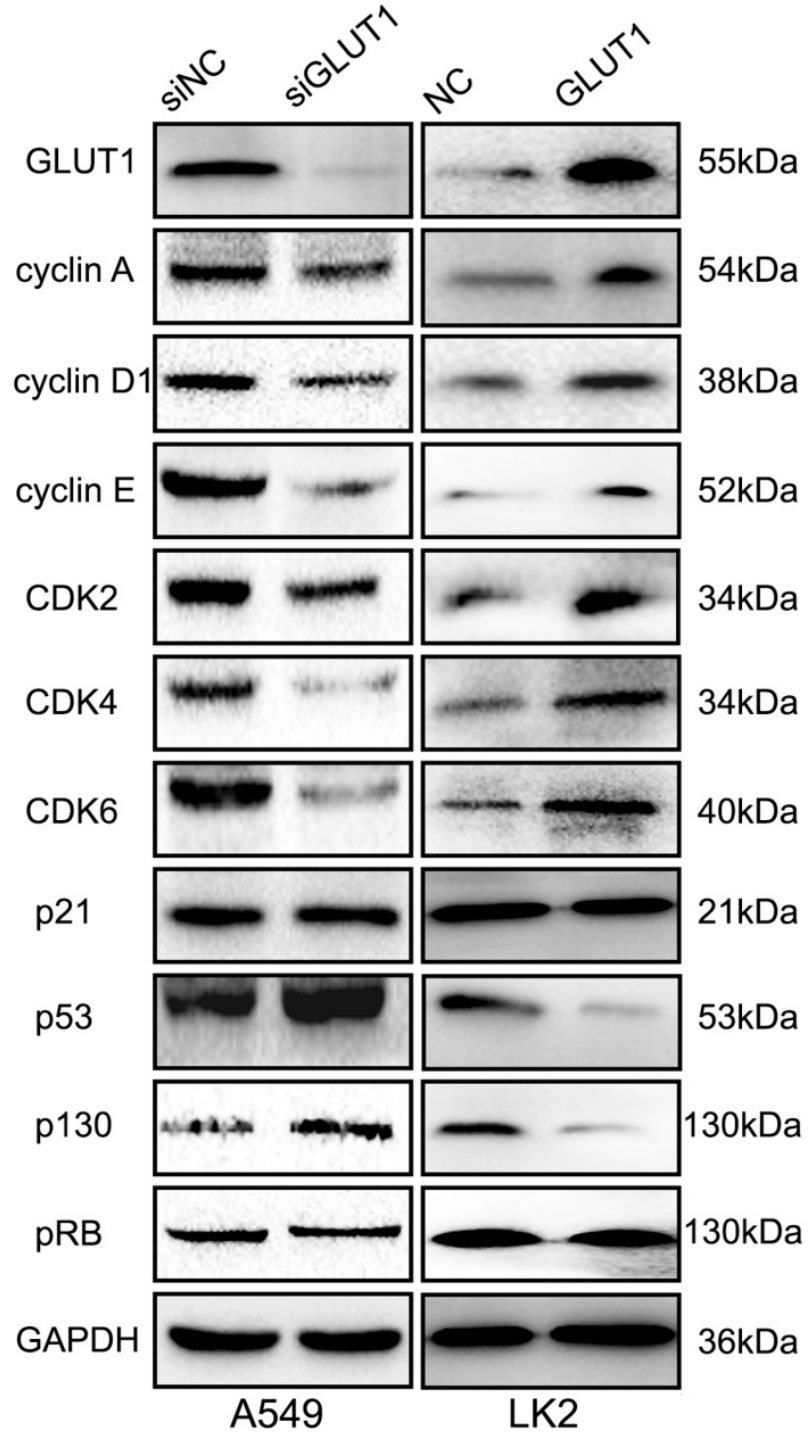

Figure 2. The effect of GLUT1 on the cell cycle correlated proteins in NSCLC cells. We added siRNA-GLUT1 (siGLUT1) to A549 cell and GLUT1 expression plasmid (GLUT1) to LK2 cell; NC, negative control; si-NC, scramble siRNA for negative control.

We used Western Blot to detect the effect of GLUT1 on integrin $\beta 1$, FAK and Src. The result showed that GLUT1 upregulated the expression of integrin $\beta 1$ and promoted the phosphorylation of FAK (Tyr576/577 phosphorylated) and Src (Tyr530 phosphorylated), but there is no effect on CD44 expression (Figure 6). After co-transfection of GLUT1 expression plasmid with the siRNA-integrin $\beta 1$, the effect of GLUT1 on cell proliferation, migration, invasion and apoptosis was significantly suppressed (Figure S3), as well as the phosphorylation of FAK (Tyr576/577 phosphorylated) and Src (Tyr530 phosphorylated) (Figure S4).

Above results indicate that GLUT1 may regulate the proliferation, migration, invasion and apoptosis of NSCLC cell through integrin $\beta 1 /$ Src/FAK signaling.

\section{Discussion}

As a member of GLUT family, GLUT1 plays an important role in Warburg effect [28]. GLUT1 overexpression is associated with the prognosis of malignant tumor patients $[29,30]$. GLUT1 expression is significantly correlated with MMP2 expression in tumor cells [11]. We found that GLUT1 was overexpressed in bronchial brushing samples of NSCLC patients, and GLUT1 expression in NSCLC tissues was higher than that in paracancerous tissues [31]. However, the potential molecular mechanism that GLUT1 regulates NSCLC cell invasion, proliferation and migration is still unclear.

We have used RT-PCR to detect GLUT1 mRNA expression of bronchial brushing specimens, which might be a useful adjunct to cytology diagnosis. It was more sensitive than cytology but its lower specificity [18]. In this study, we used quantitative real-time PCR to detect bronchial brushing samples. This method could quantitatively detect the samples, which is better than RT-PCR. Consistent with previous results, GLUT1 mRNA expression in malignant group is significantly higher than that in benign group. It has been reported that Ghrelin modulates GLUT1 expression so as to enhance oral cancer cell proliferation indirectly [19]. GLUT1 overexpression promoted cell viability and colony formation of neck squamous cell carcinoma [20]. Gene promoter methylation is associated with high-grade tumor [32], and GLUT1 promotor methylation was related with survival rate of tumor cells [33]. It presents us with a hypothesis that GLUT1 might have an effect on NSCLC cell proliferation. We transfected siRNA-GLUT1 to A549 cell and found that cell proliferation and colony formation was significantly inhibited $(P<0.05)$, as well as the expressions of cyclin D1, cyclin E, CDK2, CDK4 and CDK6, but the expressions of p53 and p130 were enhanced, as well as cell apoptosis. GLUT1 transfection to LK2 cell had the opposite effect. The phases of the cell cycle are regulated by many factors. The G1 phase is mainly mediated by CDK2-cyclin E and CDK4/6-cyclin D1 complexes and the G1/S phase transition is influenced by CDK2-cyclin A complex [34-37]. 
CDK2-cyclin E and CDK4/6-cyclin D1 complexes are important for the promotion of G1/S checkpoint transition in cell cycle $[38,39]$. The transcription factor p53 is a tumor suppressor, regulating many target genes [40]. p130 is involved in cellular senescence [41]. Cellular senescence means that cell proliferation is suppressed [42]. RB phosphorylation is important for the G1 to $S$ phase transition [43]. The cyclin-dependent kinase inhibitor p21 promotes cell cycle arrest by many stimuli [44]. As a member of the cell adhesion molecule family, CD44 plays a crucial role in tumor growth and motility [45]. Based on the above reports and our results, we concluded that GLUT1 regulated NSCLC cell proliferation through cyclin D1, cyclin E, CDK2, CDK4, CDK6, p53 and p130.
It is well known that MMP2 is a marker of tumor invasion and metastasis. GLUT1 could regulate the expression of MMP2 [11]. In this study, we found that GLUT1 could significantly enhance cell migration and invasion in NSCLC cell lines, and GLUT1 could upregulate the MMP2 expression. The detailed molecular mechanism of MMP2 mediated by GLUT1 need to do further studies.

ROCK is one of the most important effector molecules in the downstream of Rho signaling [46]. ROCK proteins (ROCK1 and ROCK2) are activated by a small GTPase protein RhoA. It has been reported that RhoA/ROCK signaling pathway is involved in regulating actin cytoskeleton and promoting actin globulin contraction $[47,48]$. In this study, GLUT1 could increase the expressions of ROCK1 and ROCK2,
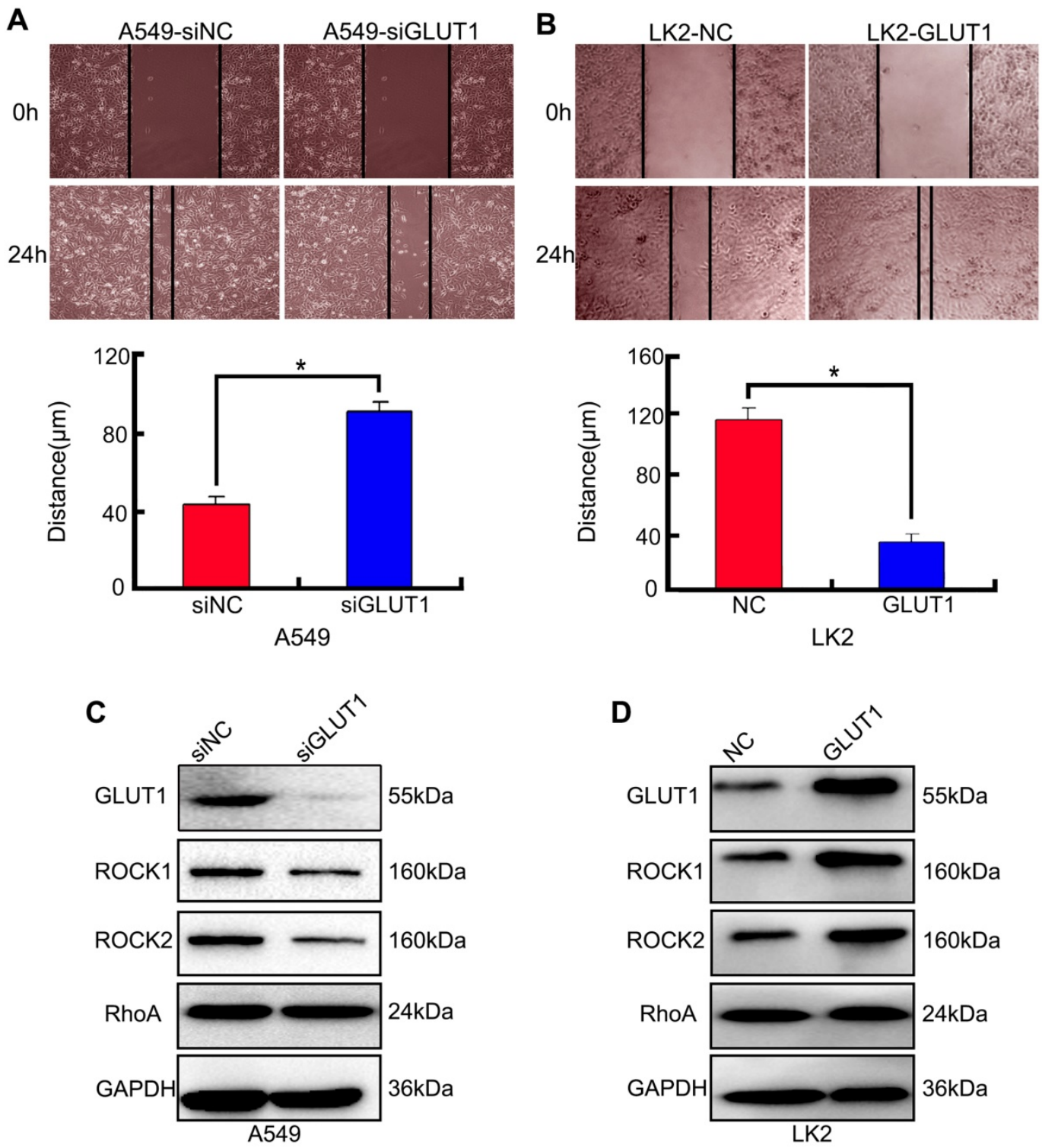

Figure 3. The effect of GLUT1 on NSCLC cell migration and correlated proteins. (A, B) NSCLC cell migration; (C, D) the expressions of ROCK1, ROCK2 and RhoA. We added siRNA-GLUT1 (siGLUT1) to A549 cell and GLUT1 expression plasmid (GLUT1) to LK2 cell; the graph in (A) and (B) shows the distance of cell migration under different treatments; *, P<0.05 compared with negative control; NC, negative control; si-NC, scramble siRNA for negative control; Error bars, S.D. 
but have not effect on RhoA. So the regulation of the NSCLC cell migration by GLUT1 might not be mediated by RhoA/ROCK signaling.

Integrins are cell surface transmembrane receptors that identify and interact with ECM proteins, playing important roles in activating signaling pathways related to cytoskeleton [49]. Focal adhesion kinase (FAK) is a non-receptor kinase that takes part in cell migration and proliferation [13-15]. The interaction of FAK with integrin $\beta 1$ is required for FAK activation [27]. Integrin-linked FAK exhibited tyrosine phosphorylation and significantly associated with Src through their SH2 domains [50]. Src is a critical mediator of FAK-regulated migratory and invasive activity [51]. In NSCLC cell, GLUT1 could upregulate the expressions of integrin $\beta 1$, Src and FAK, as well as p-Src (Tyr 530 phosphorylated) and p-FAK (Tyr576/577 phosphorylated). These results showed that phosphorylated Src and FAK was important for the regulation of the NSCLC cell migration and invasion by GLUT1. Above results indicate that GLUT1 may regulate the progression of NSCLC through integrin $\beta 1 /$ Src/FAK signaling.

B

A A549-siNC A549-siGLUT1
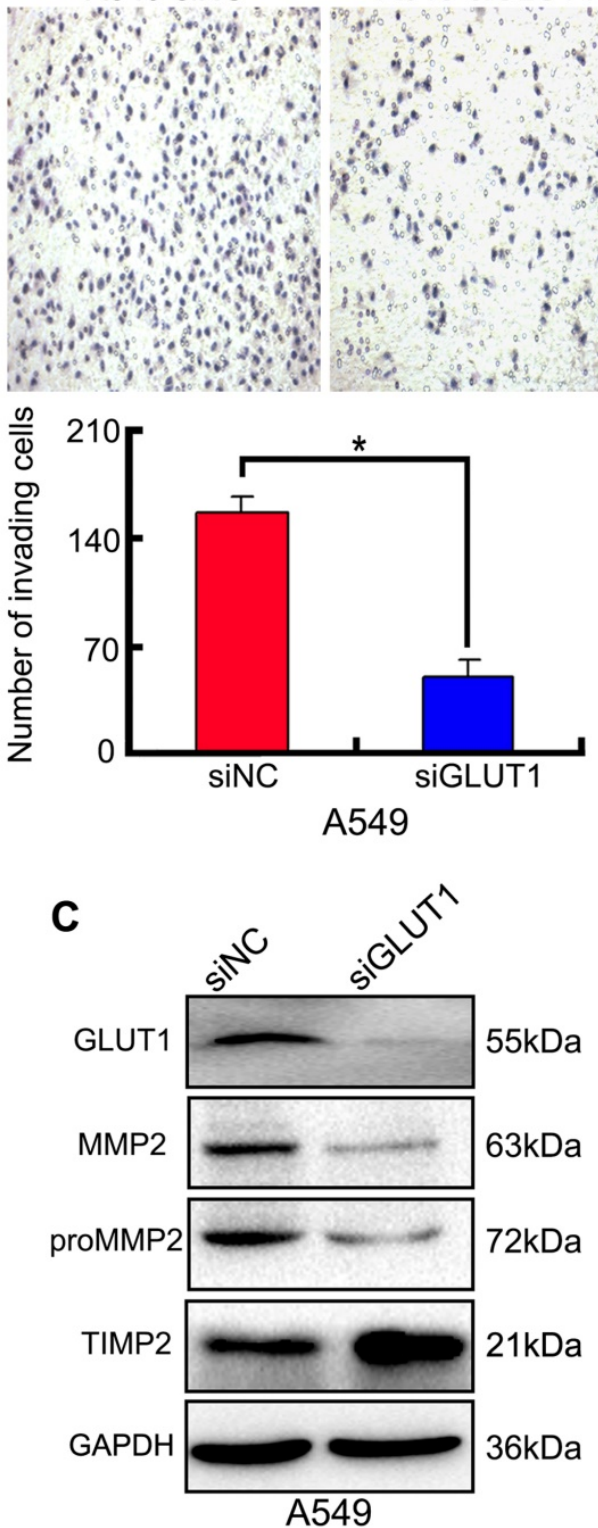

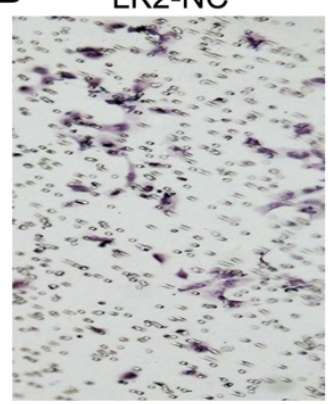

LK2- GLUT1
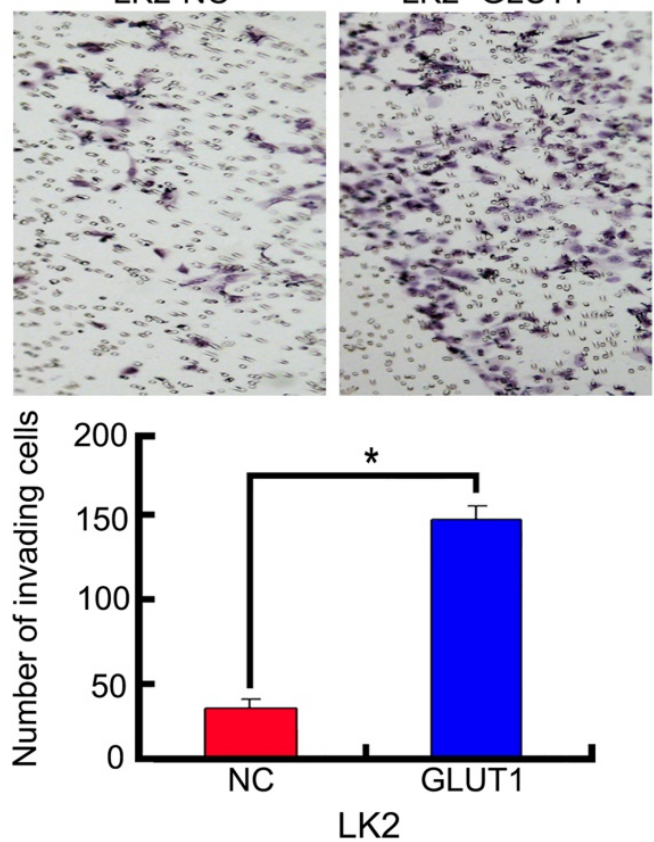

D

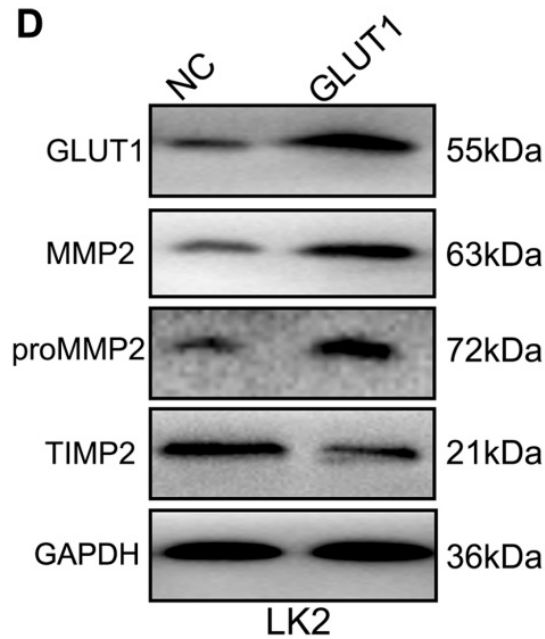

Figure 4. The effect of GLUT1 on NSCLC cell invasion and correlated proteins. (A, B) NSCLC cell invasion; (C, D) the expressions of MMP2, proMMP2 and TIMP2. We added siRNA-GLUT1 (siGLUT1) to A549 cell and GLUT1 expression plasmid (GLUTI) to LK2 cell; the graph in (A) and (B) shows the number of invading cells under different treatments; ", $P<0.05$ compared with negative control; NC, negative control; si-NC, scramble siRNA for negative control; Error bars, S.D. 

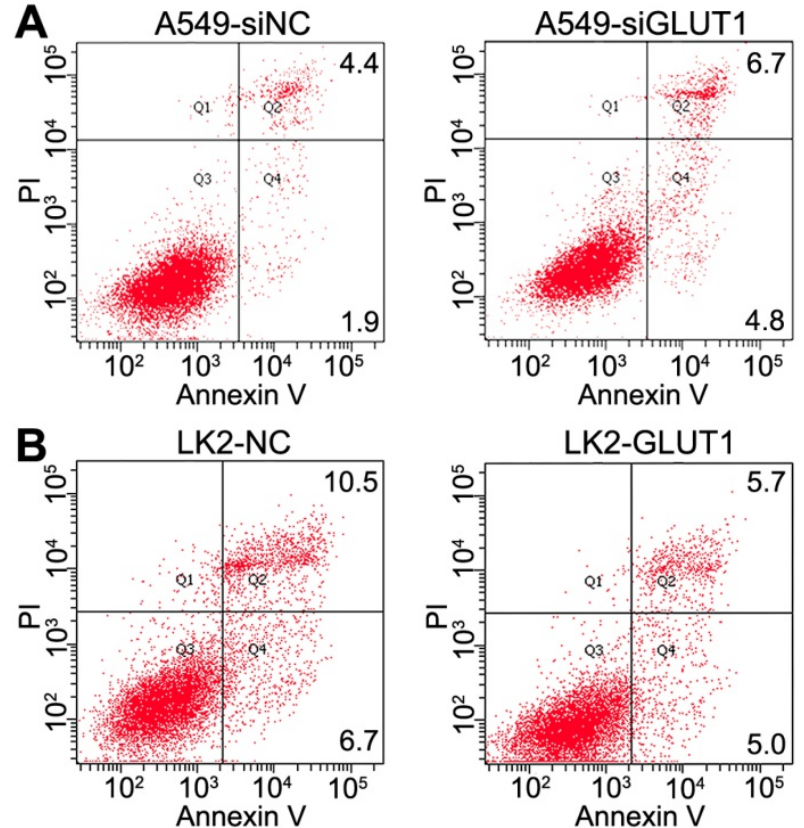

Figure 5. The effect of GLUT1 on the apoptosis in A549 and LK2 cells. We added siRNA-GLUT1 (siGLUT1) to A549 cell (A) and GLUT1 expression plasmid (GLUT1) to LK2 cell (B); NC, negative control; si-NC, scramble siRNA for negative control.

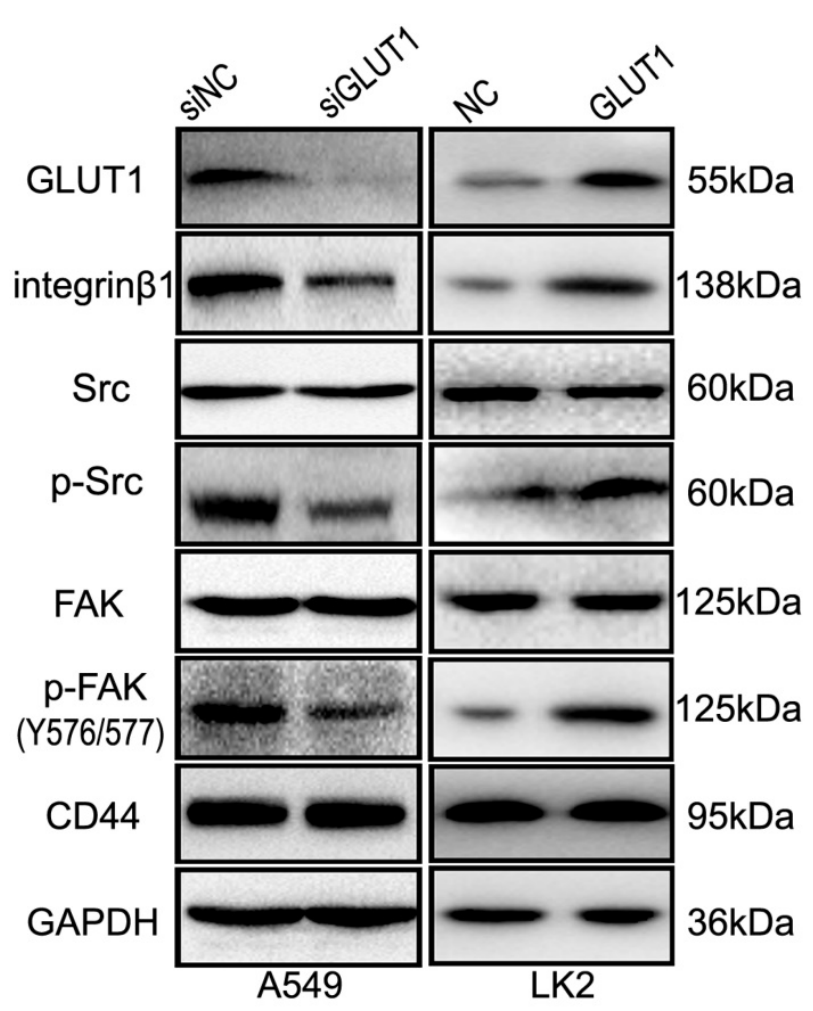

Figure 6. The effect of GLUT1 on integrin $\beta 1 / \mathrm{Src} / \mathrm{FAK}$ signaling in NSCLC cells. We added siRNA-GLUT1 (siGLUT1) to A549 cell and GLUT1 expression plasmid (GLUT1) to LK2 cell; NC, negative control; si-NC, scramble siRNA for negative control.

Integrin $\beta 1$ has been reported to play an important role in regulating cell migration, proliferation and invasion, which could activate down-stream FAK/Src signaling [52-54]. So we co-transfected GLUT1 expression plasmid with the
siRNA-integrin $\beta 1$ to LK2 cell. The results showed that adding siRNA-integrin $\beta 1$ suppressed the effect of GLUT1 on cell proliferation, invasion, migration, and apoptosis. The GLUT1 induced phosphorylation of FAK (Tyr576/577 phosphorylated) and Src (Tyr530 phosphorylated) was significantly inhibited by siRNA-integrin $\beta 1$. We could conclude that GLUT1 regulates the cell biological behavior through integrin $\beta 1$.

The genetic variation of GLUT1 could be used in predicting survival of patients with NSCLC in early stage [55]. The association between positron emission tomography (PET) parameters and GLUT1 expression is an important symbol for judging prognosis in lung adenocarcinoma [56]. This is an indication that GLUT1 expression has great clinical significance for NSCLC patients. In this study, we confirm that GLUT1 mRNA expression in bronchial brushing samples of NSCLC is significantly higher than that in benign group and GLUT1 promotes the malignant phenotype of NSCLC through integrin $\beta 1 /$ Src/FAK signaling, which provides a new target for the research and treatment of lung cancer.

\section{Abbreviations}

GLUT1: glucose transporter 1; MMP: matrix metalloproteinase; ECM: extracellular matrix; NSCLC: non-small cell lung cancer; FAK: focal adhesion kinase; TIMP2: tissue inhibitor of metalloproteinases 2; CDK: cyclin dependent kinase; ROCK: Rho-associated coiled-coil containing kinase; PBS: phosphatebuffered saline; FBS: fetal bovine serum.

\section{Supplementary Material}

Supplementary figures and table.

http://www.jcancer.org/v10p4989s1.pdf

\section{Acknowledgments}

This work was supported by the National Natural Science Foundation of China (No.81171650 and 81672082 to Guangping Wu, No. 81602022 to Huanyu Zhao).

\section{Competing Interests}

The authors have declared that no competing interest exists.

\section{References}

1. Hanahan D, Weinberg RA. Hallmarks of cancer: the next generation. Cell. 2011; 144: 646-74.

2. Medina RA, Owen GI. Glucose transporters: expression, regulation and cancer. Biol Res 2002; 35: 9-26.

3. Hsu PP, Sabatini DM. Cancer cell metabolism: Warburg and beyond. Cell. 2008; 134: 703-7.

4. Zhang HL, Wang MD, Zhou X, et al. Blocking preferential glucose uptake sensitizes liver tumor-initiating cells to glucose restriction and sorafenib treatment. Cancer Lett. 2017; 388: 1-11. 
5. Szablewski L. Glucose transporters in healthy heart and in cardiac disease. Int J Cardiol. 2017; 230: 70-5

6. Koh YW, Lee SJ, Park SY. Differential expression and prognostic significance of GLUT1 according to histologic type of non-small-cell lung cancer and its association with volume-dependent parameters. Lung Cancer. 2017; 104: 31-7.

7. Sawayama H, Ishimoto $\mathrm{T}, \mathrm{Watanabe} \mathrm{M}$, et al. High expression of glucose transporter 1 on primary lesions of esophageal squamous cell carcinoma is associated with hematogenous recurrence. Ann Surg Oncol. 2014; 21: 1756-62.

8. Yu M, Zhou Q, Zhou Y, et al. Metabolic phenotypes in pancreatic cancer. PLoS One. 2015; 10: e0115153.

9. Kim S, Ahn M, Piao $\mathrm{Y}$, et al. Effect of botulinum toxin type A on TGF-beta/Smad pathway signaling: Implications for Silicone-induced capsule formation. Plast Reconstr Surg. 2016; 138: 821e-9e.

10. Noh S, Jung JJ, Jung M, et al. Body fluid MMP2 as a putative biomarker in metastatic breast cancer. Oncol Lett. 2012; 3: 699-703.

11. Ito S, Fukusato T, Nemoto T, et al. Coexpression of glucose transporter 1 and matrix metalloproteinase-2 in human cancers. J Natl Cancer Inst. 2002; 94: 1080-91.

12. Liu $Z$, Han $X$, Chen $R$, et al. Microfluidic mapping of cancer cell-protein binding interaction. ACS Appl Mater Interfaces. 2017; 9: 22143-8.

13. Lim ST, Chen XL, Lim Y, et al. Nuclear FAK promotes cell proliferation and survival through FERM-enhanced p53 degradation. Mol Cell. 2008; 29: 9-22

14. Yamamoto D, Sonoda $\mathrm{Y}$, Hasegawa M, et al. FAK overexpression upregulates cyclin D3 and enhances cell proliferation via the PKC and PI3-kinase-Akt pathways. Cell Signal. 2003; 15: 575-83.

15. Mitra SK, Hanson DA, Schlaepfer DD. Focal adhesion kinase: in command and control of cell motility. Nat Rev Mol Cell Biol. 2005; 6: 56-68

16. Liu L, Zong C, Li B, et al. The interaction between $\beta 1$ integrins and ERK1/2 in osteogenic differentiation of human mesenchymal stem cells under fluid shear stress modelled by a perfusion system. J Tissue Eng Regen Med. 2014; 8: 85-96.

17. Oh S, Kim H, Nam K, et al. Glut1 promotes cell proliferation, migration and invasion by regulating epidermal growth factor receptor and integrin signaling in triple-negative breast cancer cells. BMB Rep. 2017; 50: 132-7.

18. Lv M, Fan YB, Zhao YJ, et al. Expression and clinical significance of glucose transporter 1 mRNA in bronchialbrushing liquid-based cytology specimens from patients with and without lung cancer. Cytopathology. 2012; 23: 108-13.

19. Kraus D, Reckenbeil J, Wenghoefer M, et al. Ghrelin promotes oral tumor cell proliferation by modifying GLUT1 expression. Cell Mol Life Sci. 2016; 73: 1287-99.

20. Li S, Yang X, Wang P, et al. The effects of GLUT1 on the survival of head and neck squamous cell carcinoma. Cell Physiol Biochem. 2013; 32: 624-34.

21. Srivastava P, Lone TA, Kapoor R, et al. Association of promoter polymorphisms in MMP2 and TIMP2 with prostate cancer susceptibility in North India. Arch Med Res. 2012; 43: 117-24.

22. Seiki M. Membrane-type 1 matrix metalloproteinase: A key enzyme for tumor invasion. Cancer Lett. 2003; 194: 1-11.

23. Case LB, Waterman CM. Integration of actin dynamics and cell adhesion by a threedimensional, mechanosensitive molecular clutch. Nat Cell Biol. 2015; 17: 955-63.

24. Maartens AP, Brown NH. Anchors and signals: the diverse roles of integrins in development. Curr Top Dev Biol. 2015; 112: 233-72.

25. Wolfenson H, Lavelin I, Geiger B. Dynamic regulation of the structure and functions of integrin adhesions. Dev Cell. 2013; 24: 447-58.

26. Yu W, Gowda M, Sharad Y, et al. Oxidation of KCNB1 potassium channels triggers apoptotic integrin signaling in the brain. Cell Death Dis. 2017; 8: e2737.

27. Zhao XK, Cheng Y, Liang Cheng M, et al. Focal adhesion kinase regulates fibroblast migration via integrin beta- 1 and plays a central role in fibrosis. Sci Rep. 2016; 6: 19276.

28. Zhang C, Liu J, Liang $Y$, et al. Tumour-associated mutant p53 drives the Warburg effect. Nat Commun. 2013; 4: 2935.

29. Sun HW, Yu XJ, Wu WC, et al. GLUT1 and ASCT2 as predictors for prognosis of hepatocellar carcinoma. PLoS One. 2016; 11: e0168907.

30. Wellberg EA, Johnson S, Finlay-Schultz J, et al. The glucose transporter GLUT1 is required for ErbB2-induced mammary tumorigenesis. Breast Cancer Res. 2016; 18: 131

31. Fan R, Hou WJ, Zhao YJ, et al. Overexpression of HPV16 E6/E7 mediated HIF-1a upregulation of GLUT1 expression in lung cancer cells. Tumour Biol. 2016; 37: 4655-63.

32. Fu LN, Wang YQ, Tan J, et al. Role of JMJD2B in colon cancer cell survival under glucose-deprived conditions and the underlying mechanisms. Oncogene. 2018; 37: 389-402.

33. Giannopoulou L, Chebouti I, Pavlakis K, et al. RASSF1A promoter methylation in high-grade serous ovarian cancer: A direct comparison study in primary tumors, adjacent morphologically tumor cell-free tissues and paired circulating tumor DNA. Oncotarget. 2017; 8: 21429-43.

34. Kim TH, Oh S, Kim SS. Recombinant human prothrombin kringle-2 induces bovine capillary endothelial cell cycle arrest at G0-G1 phase through inhibition of cyclin D1/CDK4 complex: modulation of reactive oxygen species generation and up-regulation of cyclin-dependent kinase inhibitors. Angiogenesis. 2005; 8: 307-14

35. Zhu Y, Wei $\mathrm{W}$, Ye $\mathrm{T}$, et al. Small molecule $\mathrm{TH}-39$ potentially targets Hec1/Nek2 interaction and exhibits antitumor efficacy in K562 cells via G0/G1 cell cycle arrest and apoptosis induction. Cell Physiol Biochem. 2016; 40: $297-308$
36. Na K, Li K, Sang T, et al. Anticarcinogenic effects of water extract of sporoderm-broken spores of Ganoderma lucidum on colorectal cancer in vitro and in vivo. Int J Oncol. 2017; 50: 1541-54.

37. Miele A, Braastad CD, Holmes WF, et al. HiNF-P directly links the cyclin $\mathrm{E} / \mathrm{CDK} 2 / \mathrm{p} 220 \mathrm{NPAT}$ pathway to histone $\mathrm{H} 4$ gene regulation at the G1/S phase cell cycle transition. Mol Cell Biol. 2005; 25: 6140-53.

38. Huang $\mathrm{Y}, \mathrm{Wu} \mathrm{G}$, Fan $\mathrm{H}$, et al. Electroacupuncture promotes chondrocyte proliferation via accelerated G1/S transition in the cell cycle. Int J Mol Med. 2013; 31: 1443-8

39. Frade JM, Ovejero-Benito MC. Neuronal cell cycle: the neuron itself and its circumstances. Cell Cycle. 2015; 14: 712-20.

40. Zhu L, Lu Z, Zhao H. Antitumor mechanisms when $\mathrm{pRb}$ and $\mathrm{p} 53$ are genetically inactivated. Oncogene. 2015; 34: 4547-57.

41. Fiorentino FP, Symonds CE, Macaluso M, et al. Senescence and p130/Rbl2: a new beginning to the end. Cell Res. 2009; 19: 1044-51.

42. Kuilman T, Michaloglou C, Mooi WJ, et al. The essence of senescence. Genes Dev. 2010; 24: 2463-79.

43. Massagué J. G1 cell-cycle control and cancer. Nature. 2004; 432: 298-306.

44. Abbas T, Dutta A. p21 in cancer: intricate networks and multiple activities. Nat Rev Cancer. 2009; 9: 400-14.

45. Park GB, Ko HS, Kim D. Sorafenib controls the epithelial-mesenchymal transition of ovarian cancer cells via EGF and the CD44-HA signaling pathway in a cell type-dependent manner. Mol Med Rep. 2017; 16: 1826-36.

46. Shimizu T, Liao JK. Rho Kinases and cardiac remodeling. Circ J. 2016; 80: $1491-8$

47. Ruiz-Loredo AY, López E, López-Colomé AM. Thrombin promotes actin stress fiber formation in RPE through Rho/ROCK-mediated MLC phosphorylation. J Cell Physiol. 2011; 226: 414-23.

48. Jordan SN, Canman JC. Rho GTPases in animal cell cytokinesis: an occupation by the one percent. Cytoskeleton (Hoboken). 2012; 69: 919-30.

49. Legate KR, Wickström SA, Fässler R. Genetic and cell biological analysis of integrin outside-in signaling. Genes Dev. 2009; 23: 397-418.

50. Schaller MD, Hildebrand JD, Shannon JD, et al. Autophosphorylation of the focal adhesion kinase, pp125FAK, directs SH2-dependent binding of pp60src. Mol Cell Biol. 1994; 14: 1680-8.

51. Kong DB, Chen F, Sima N. Focal adhesion kinases crucially regulate TGF $\beta$-induced migration and invasion of bladder cancer cells via Src kinase and E-cadherin. Onco Targets Ther. 2017; 10: 1783-92.

52. Lee HD, Kim YH, Kim DS. Exosomes derived from human macrophages suppress endothelial cell migration by controlling integrin trafficking. Eur J Immunol. 2014; 44: 1156-69.

53. Miao L, $\mathrm{X}$ in $\mathrm{X}, \mathrm{X}$ in $\mathrm{H}$, et al. Hydrogen sulfide recruits macrophage migration by integrin $\beta 1$-Src-FAK/Pyk2-Rac pathway in myocardial infarction. Sci Rep. 2016; 6: 22363.

54. Penchev VR, Rasheed ZA, Maitra A, et al. Heterogeneity and targeting of pancreatic cancer stem cells. Clin Cancer Res. 2012; 18: 4277-84.

55. Do SK, Jeong JY, Lee SY, et al. Glucose transporter 1 gene variants predict the prognosis of patients with early-stage non-small cell lung cancer. Ann Surg Oncol. 2018; 25: 3396-403.

56. Koh YW, Park SY, Hyun SH, et al. Associations between PET textural features and GLUT1 expression, and the prognostic significance of textural features in lung adenocarcinoma. Anticancer Res. 2018; 38: 1067-71 